\title{
ARTICLE \\ HPA axis response and psychosocial stress as interactive predictors of suicidal ideation and behavior in adolescent females: a multilevel diathesis-stress framework
}

\author{
Tory A. Eisenlohr-Moul ${ }^{1}$, Adam B. Miller ${ }^{2}$, Matteo Giletta ${ }^{3}$, Paul D. Hastings ${ }^{4}$, Karen D. Rudolph ${ }^{5}$, Matthew K. Nock ${ }^{6}$ and \\ Mitchell J. Prinstein ${ }^{2}$
}

Prior studies suggest that hypothalamic-pituitary-adrenal (HPA) axis stress response is a relatively stable risk factor for suicidal behavior in adults, and also that fluctuations in developmentally salient social stress (i.e., peer stress) predict acute suicidal ideation and behavior in adolescent females. Here, we test the hypothesis that periods of high peer stress are prospectively associated with concurrent episodes of suicidal ideation and behavior, but that abnormal cortisol responses to stress at baseline act as a diathesis that strengthens this stressor-suicidality link, using a longitudinal, multi-wave study design. We measured cortisol responses to an adolescent modification of the Trier Social Stress Test in 220 adolescent females recruited for mental health concerns, and measured stress (peer, academic, and mother-child) and suicidal ideation and behaviors repeatedly across 18 months. Logistic multilevel models revealed the predicted interaction between altered cortisol response and elevated periods of peer stress in predicting suicidal behavior, but not ideation. Higher-than-usual peer stress predicted suicidal ideation regardless of HPA function, but predicted suicide attempt only among those with blunted cortisol responses. Results suggest that peer stress serves to trigger suicidal ideation among female youth, but only triggers suicidal behavior among those with blunted cortisol reactivity.

Neuropsychopharmacology (2018) 43:2564-2571; https://doi.org/10.1038/s41386-018-0206-6

\section{INTRODUCTION}

Suicide rates in the US increased markedly from 1999 to 2014, especially among adolescent females [1]. Most models of suicidal thoughts and behavior propose that they arise due to dynamic interactions between stable risk factors (e.g., biological diatheses) and acute environmental stressors; however, such models are rarely tested. The present study examines the dynamic interplay between a biological diathesis (altered hypothalamic-pituitary-adrenal (HPA) axis stress response at baseline) and fluctuations in psychosocial stress states in the development of acute adolescent suicidality across 18 months.

Developmentally salient psychosocial stress and suicide risk Diathesis-stress models of suicide often emphasize precipitating roles of developmentally salient stressors; female adolescents experience normative increases in sensitivity to social evaluation that may serve as triggers for suicidal behavior among at-risk individuals. Indeed, psychosocial stress in one's peer group (bullying, social rejection, conflict) frequently precede the onset of suicidal ideation and behavior among adolescent females [2-6], although the specificity of peer stress (vs. academic or parental stress) as a precipitant has never been fully tested. While normative increases in psychosocial sensitivity occur, most individuals do not experience suicidal ideation or behavior during episodes of peer stress. Therefore, it is critical to identify vulnerability factors determining which individuals are at risk for suicidal ideation or behavior in the context of developmentally salient stressors.

HPA axis activation and suicide risk

Given its well-defined role in adaptive responses to environmental stressors, the HPA axis stress response represents a relatively stable factor $[7,8]$ that may shape the influence of developmentally salient stressors (i.e., peer stress) on suicidal ideation and behavior in adolescent females. Strong evidence links altered HPA axis function to suicidal ideation and behavior, although much of this evidence is in adults and all studies were cross-sectional. The one study examining the HPA axis and suicidal ideation among adolescent females $(N=138)$ draws from an early subsample from the present study; both hyperreactivity and hyporeactivity (blunting) of cortisol (CORT) response to a psychosocial stressor predicted suicidal ideation over a 3-month period, although the association with blunting was marginally significant [9]. Two adult studies $(N=160,208)$ demonstrated blunted CORT to psychosocial stress among those with suicidal behavior relative to controls or those with suicidal ideation only $[10,11]$. Two studies have also found blunted Trier Social Stress Test (TSST) CORT response among adolescent females with non-suicidal self-injury, which

${ }^{1}$ Department of Psychiatry, University of Illinois at Chicago, 912 S. Wood Street, South Tower, Room 335, Chicago, IL, United States; ${ }^{2}$ Department of Psychology and Neuroscience,

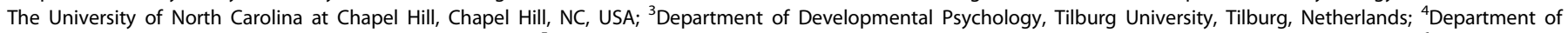

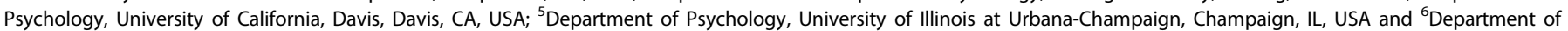
Psychology, Harvard University, Cambridge, MA, USA

Correspondence: Tory A. Eisenlohr-Moul (temo@uic.edu)

Received: 15 November 2017 Revised: 25 April 2018 Accepted: 19 August 2018

Published online: 6 September 2018 
often precedes suicidal behaviors [12, 13]. Several psychosocial correlates of suicidal ideation and behavior predict altered HPA axis profiles in youth: depressive disorder predicts hyperreactive CORT response $[14,15]$, and blunted CORT responses are observed in both trauma histories $[16,17]$ and impulse-control disorders $[18$, 19].

In sum, altered CORT responses (blunting and hyperresponse) have been linked to depression and suicidal ideation, whereas blunted CORT responses have been found in NSSI and suicidal behavior. Moderate CORT response facilitates cognitive function during stress [20] and serves to limit the intensity and duration of stress responses across multiple systems (e.g., immune, sympathetic). Therefore, moderate CORT stress responses may facilitate coping [21] whereas hypo- or hyper-responsiveness of the HPA axis may increase vulnerability to suicide during periods of stress [10]. This study is the first to directly examine this diathesis-stress perspective, modeling the dynamic interplay between a biological diathesis (i.e., altered HPA axis response to stress) and a developmentally salient stressor (i.e., peer stress vs. academic or mother-child stress) using a novel, prospective (18 months) multilevel framework in adolescent females $(N=220)$. Although adult males comprise the majority of subjects in previouslypublished research on the role of altered HPA axis function in suicidal behavior, we predict that HPA axis dysregulation will similarly confer risk for suicidal ideation and behavior in this adolescent female sample. We hypothesized that peer stress triggers increased risk of ideation and behavior specifically among adolescent females with altered CORT stress responses. Specifically, we predicted an interaction between the baseline area under the TSST CORT curve with respect to increase $\left(A U C_{i}\right.$; between-person factor) and fluctuations in peer stress (withinperson factor) predicting concurrent suicidal ideation and behavior. We expected that higher-than-usual peer stress predicts concurrent suicidal ideation and behavior, but only among those with either blunted or hyperreactive CORT $A U C_{i}$.

\section{MATERIALS AND METHODS}

Procedures were approved by the institutional review board. Data were collected during 2010-2015.

\section{Sample}

Subjects were 220 female adolescents (age $M=14.69, \mathrm{SD}=1.37$, range $=12-16$ ), reporting a history of at least one mental health concern in the past 2 years. Since suicidal ideation and behavior cuts across diagnostic categories, various recruitment methods were used to enroll a transdiagnostic sample enriched for risk of suicidal ideation and behavior: local inpatient units $(33 \%$ of sample; $51 \%$ of eligible subjects enrolled), advertising in outpatient clinical practices ( $12 \%$ of sample; $70 \%$ of eligible subjects enrolled), advertisements ( $40 \%$ of sample; $65 \%$ of eligible subjects enrolled), and emails to university employees (15\% of sample; $58 \%$ of eligible subjects enrolled). Inclusion criteria were: (a) female sex; (b) age 12-16 years; (c) caregiver willing to participate; (d) caregiver-reported history of a mental health concern in the past 2 years. Exclusion criteria were: psychosis, intellectual disability, or other developmental disorder. Subjects were born mostly (92\%) in the US; $24 \%$ identified as African American, $64 \%$ as White, $10 \%$ as mixed/other, $1 \%$ as Asian American, and $1 \%$ as Latino/a. Six percent identified as Hispanic. As a proxy for socioeconomic status, we measured highest guardian education: $1.3 \%$ reported some high school; $13.3 \%$ high school or GED; $12.8 \%$ trade degree; $17.8 \%$ some college; $24.7 \%$ bachelor's degree; $11 \%$ some graduate school; $13.3 \%$ master's degree; $5.5 \%$ doctoral degree. Forty-one percent of girls reported a history of sexual or physical abuse; $23 \%$ reported a history of physical abuse; and $29 \%$ reported a history of sexual abuse (18\% sexual abuse only; $12 \%$ physical abuse only; $11 \%$ both physical and sexual abuse). The mean number of PTSD symptoms endorsed on the MINI-KID was 2.68 (SD = 3.65). During recruitment, caregivers provided reports of adolescent symptoms using the Behavioral Assessment System for Children (BASC-PRS); [22]: $20 \%$ had clinically elevated $T$ scores (female, age-normed scores equal to or higher than 70) on the conduct disorder scale (raw score $M=5.92, \mathrm{SD}=4.27$ ), $21 \%$ on the attention problem scale (raw score $M=9.40, S D=4.40$ ), $17 \%$ on the hyperactivity scale (raw score $M=7.69, \mathrm{SD}=5.16$ ), $11 \%$ on the anxiety scale (raw score $M=8.89, \mathrm{SD}=5.00$ ), and $10 \%$ on the depression scale (raw score $\mathrm{M}=8.65, \mathrm{SD}=4.93$ ).

TSST and CORT sampling, assay, and coding

During a baseline laboratory visit, subjects completed a modified adolescent version of the Trier Social Stressor Test (TSST; see [9]), a standard psychosocial stressor that produces robust CORT responses [23]. Subjects provided saliva using the Sarstedt Salivette Synthetic Swab (Sarstedt, Newton, NC 28658, USA) at 5 time points: (1) upon arrival, (2) pre-TSST baseline (after $2.5 \mathrm{~h}$ in the laboratory, following interview and self-report measures; immediately before TSST instructions), (3) $20 \mathrm{~min}$ after the TSST, (4) $30 \mathrm{~min}$ after the TSST, and (5) $40 \mathrm{~min}$ after the TSST. No stressful procedures were administered for $30 \mathrm{~min}$ before the baseline sample, or for $40 \mathrm{~min}$ after the speech. Salivary samples were frozen/stored at $-25^{\circ} \mathrm{C}$ and shipped on dry ice to Salimetrics for assay using EIA. EIA kits have excellent lower limit sensitivity, ranging from $0.007 \mu \mathrm{g} / \mathrm{dL}$ to $1.2 \mu \mathrm{g} / \mathrm{mL}$. CORT values at each time point were log transformed prior to the AUC calculation to improve normality and reduce the impact of outliers.

We observed the typical CORT stress response to the TSST, with significant increases over baseline at $20 \mathrm{~min}$ post-TSST (Estimate $=0.03, \mathrm{SE}=0.005, t(860)=5.96, p<0.0001)$, and $30 \mathrm{~min}$ post-TSST (Estimate $=0.011, \mathrm{SE}=0.005, t(860)=2.15, p=0.03$ ), with a return to baseline at $40 \mathrm{~min}$ post-TSST (Estimate $=-0.001, \mathrm{SE}=$ $0.005, t(860)=0.20, p=0.84)$. Following methods set forth by Pruessner et al. [24], we plotted a reactivity curve for each subject, calculating area under the curve (AUC) with respect to increase $\left(A \cup C_{i}\right) . A U C_{i}$ represents a reactivity index reflecting both the peak and duration of HPA response. This $A U C_{i}$ was calculated using the final four samples. Because the preponderance of work on the HPA axis and suicide implicates cortisol reactivity to psychosocial stress, and because our hypotheses focus on the stress response rather than overall HPA axis tone, we focus on $A U C_{i}$ (i.e., reactivity to the stressor) rather than $A U C_{g}$ (i.e., total output). However, $A U C_{g}$ was also calculated and substituted for $A U C_{i}$ in specificity analyses.

\section{Assessment}

At baseline, the subject and a caregiver completed an initial laboratory visit. After providing informed consent/assent, adolescents and their caregiver underwent clinical interviews separately. Covariates (age, psychotropic use, oral contraceptive use) were assessed via self-report from both adolescents and their caregiver. The Self-Injurious Thoughts and Behaviors Interview (SITBI [25]) measured the presence (1) or absence (0) of suicidal ideation ("Have you ever had thoughts of killing yourself?"), and suicidal behavior, which included suicide attempt ("Have you ever made an actual suicide attempt with at least some intent to die?") and aborted or interrupted suicide attempt ("Have you ever been very close to killing yourself and at the last minute someone or something else stopped you?"). The MINI-KID Psychiatric Interview [26] measured lifetime physical or sexual abuse. Adolescents completed questionnaires, including the Moods and Feelings Questionnaire indexing depression (MFQ [27]) and the Child Chronic Strain Questionnaire (CCSQ [28]), providing stress indices for several domains (in the present study, we focus on peer stress, mother-child stress, and academic stress). Next, the modified TSST was administered and CORT response was measured in saliva.

Following this laboratory visit, follow-up calls occurred at 3,6, 9, 12,15 , and 18 months post-baseline. Follow-up calls included 
repeated measures of stress in the prior 3 months using the CCSQ and a shortened SITBI to evaluate the presence of suicidal ideation and behavior in the past 3 months. Stress subscale scores were used as both trait and state measures. Indices of trait stress in each domain as well as trait depression (Level 2) were calculated as the mean of self-reports for a given subject (baseline and all followup), standardized according to the full sample. Within-person fluctuations in (Level 1) stress were calculated as the score at a given time point minus a subject-specific mean for the variable (the Level 2 mean), divided by the subject-specific standard deviation across all visits (i.e., person-standardized).

Statistical analyses

We used logistic multilevel models in SAS PROC GLIMMIX to test whether continuous measures of cortisol reactivity $\left(A \cup C_{i}\right.$, with both rectilinear and quadratic terms) moderated the withinperson association between stress domains and risk of suicidal ideation or behavior at follow-up time points. Current dichotomous suicidal ideation or behavior were modeled as separate outcomes in log-link, binary outcome multilevel models. Repeated observations (baseline, 3, 6, 9, 12, 15, and 18 months) were nested within adolescents. Models were fit assuming incomplete data as missing completely at random (MCAR; e.g., [29, 30]). Little's [30] MCAR test suggested subjects with and without missing data did not differ significantly, $x^{2}(342)=377.87 ; p=0.09$. Missingness for repeated measures at each follow-up time point was as follows: $9 \%$ at 3 months, $12 \%$ at 6 months, $9 \%$ at 9 months, $16 \%$ at 12 months, $16 \%$ at 15 months, and $20 \%$ at 18 months.

For both outcomes separately (ideation and behavior), we predicted the outcome at a given time point from CORT $A U C_{i}$, current within-person fluctuations in a stress domain, and their interaction. Three separate models examined each domain. All models included a priori covariates: baseline presence or absence of the outcome, age (between-person factor), visit timing (hour of the day; between-person factor), time elapsed since initial visit (within-person factor), oral contraceptive use (between-person factor), psychotropic use (between-person factor), corticosteroid use (between-person factor), sample-standardized mean depression (between-person factor), and sample-standardized mean stress in a given domain (between-person factor). Models predicting suicidal behavior also included an additional dichotomous within-person covariate indicating the current presence (1) or absence (0) of suicidal ideation; this covariate allows for examining whether predictors explain variance in suicidal behavior above and beyond associations with current suicidal ideation.

Random intercepts and slopes of stress predictors were included when doing so improved model fit $(-2$ pseudo-loglikelihood); inclusion of all random effects did not substantively alter results. Because current stress was person-standardized, gamma coefficients and associated odds ratios are interpreted as the effect of a one subject-specific standard deviation change in the predictor relative to the subject-specific mean.

Power

Using G*Power [31] and multilevel adjustment methods [32], posthoc sensitivity analysis determined the smallest detectible odds ratio for suicidal behavior (the rarer outcome) across follow-up using logistic regression. Given the intraclass correlation (ICC) for suicidal behavior $(I C C=0.15)$ and our sample size $(L 2 N=$ 220 subjects, $\mathrm{L} 1 \mathrm{~N}=1093$ assessments; adjusted $N=685$ assessments), the smallest detectible odds ratio for any single predictor (given $R^{2}$ other $X=0.25$, power $=0.8$, and $a=0.05$ ) was $1.36-a$ conventionally small effect. We also conducted post-hoc sensitivity analysis for the descriptive CORT analyses described below, examining the interaction between suicide group (no history, ideation history, attempt history) and TSST time point in predicting CORT. Sensitivity analyses (CORT ICC $=0.54$; L2N $=$ 220 subjects; $\mathrm{L} 1 \mathrm{~N}=1205$ assessments; adjusted $N=352$ assessments; power $=0.8 ; a=0.05$; nonsphericity correction $=$ 0.75 ) indicated that the smallest detectible effect size was $f=$ 0.087-a conventionally small effect.

\section{RESULTS}

Descriptive analyses

Rates of suicidal ideation at each time point were relatively high, reflecting the clinical enrichment of our sample: $49.4 \%$ at baseline, $25.6 \%$ at 3 months, $19.1 \%$ at 6 months, $20.1 \%$ at 9 months, $18.8 \%$ at 12 months, $13.6 \%$ at 15 months, and $18.5 \%$ at 18 months. Rates of suicidal behavior at each time point were lower but also elevated: $33.6 \%$ at baseline, $8.4 \%$ at 3 months, $6.2 \%$ at 6 months, $2.5 \%$ at 9 months, $6.5 \%$ at 12 months, $2.2 \%$ at 15 months, and $2.9 \%$ at 18 months (see [33] for additional information). Bivariate associations among study variables are reported in Supplement 1; notably, neither abuse exposure nor the number of PTSD symptoms endorsed was significantly related to CORT $A U C_{i}$.

Descriptive multilevel models using the repeated measures TSST log CORT data were used to compare TSST CORT response in three suicide groups (category-coded based on all information collected at baseline and follow-ups: no lifetime ideation or behavior, $n=53$; any lifetime suicidal ideation but not behavior, $n$ $=81$; any lifetime suicidal behavior, $n=85$ ). No significant group differences on baseline CORT were observed. Individuals with lifetime suicidal behavior demonstrated blunted CORT response across the four TSST samples compared with those without lifetime ideation or behavior (Interaction Estimate at Peak = $-0.035, \mathrm{SE}=0.016, t(682)=-2.11, p=0.035)$ and also showed blunting relative to those reporting a lifetime history of ideation but not behavior (Interaction Estimate at Peak $=-0.027$, SE $=$ $0.013, t(689)=-2.07, p=0.038)$. There were no significant differences in response between those with no history of suicidality and those with suicidal ideation but not behavior, (Interaction Estimate at Peak $=-0.012, \mathrm{SE}=0.012, t(682)=-1.01$, $p=0.31$ ). See Fig. 1 for means plots for CORT across the TSST by group. In sum, although no baseline (pre-TSST) CORT differences were observed across groups, blunting of the stress response at peak TSST stress was observed in those reporting any suicidal behavior relative to those with no history of any suicidality, and relative to those with ideation but not behavior (Table 1).

\section{Hypothesis tests}

Our diathesis-stress model hypothesizes a cross-level interaction between fluctuations in real-life peer stress and TSST CORT psychosocial stress response in the prediction of concurrent suicidal ideation and behaviors. We predicted either blunted or hyperreactive CORT $A U C_{i}$ to the TSST would strengthen the

\section{Cortisol Across the TSST by Suicide Group}

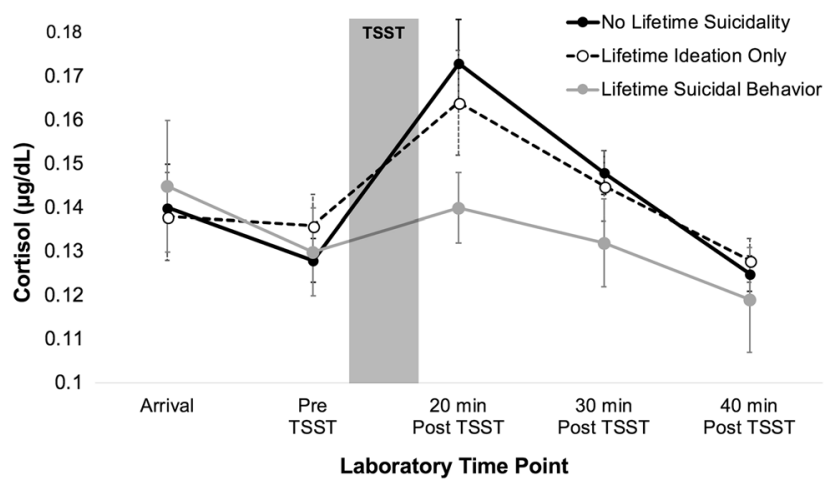

Fig. 1 Means and standard errors for log CORT ( $\mu \mathrm{g} / \mathrm{dL})$ values across the TSST by lifetime suicide status 
Table 1. Summary of intercorrelations and descriptive statistics for continuous between-person variables $(N=220)$

\begin{tabular}{|c|c|c|c|c|c|c|c|c|}
\hline Variable & 1 & 2 & 3 & 4 & 5 & 6 & 7 & 8 \\
\hline \multicolumn{9}{|l|}{ 1. Age } \\
\hline 2. Log TSST CORT AUC & 0.01 & & & & & & & \\
\hline 4. Mean Peer Stress & $0.16^{*}$ & -0.05 & $0.63^{* * *}$ & & & & & \\
\hline 5. Mean Mother-Child Stress & 0.12 & -0.07 & $0.35^{* * *}$ & $0.34^{* * *}$ & & & & \\
\hline 8. \# Suicidal Behavior Reports & $0.23^{* * *}$ & $-0.13^{*}$ & $0.43^{* * *}$ & $0.43^{* * *}$ & $0.17^{*}$ & $0.23^{* * *}$ & $0.65^{* * *}$ & \\
\hline$N$ & 220 & 218 & 220 & 220 & 220 & 220 & 220 & 220 \\
\hline Mean & 14.67 & 2.79 & 0.43 & 2.01 & 2.16 & 2.23 & 1.5 & 0.58 \\
\hline Standard deviation & 1.36 & 6.20 & 0.29 & 0.55 & 0.71 & 0.75 & 1.8 & 0.94 \\
\hline Range & $12-16$ & -13.60 to 27.13 & $0-1.57$ & $1-4.90$ & $1-5$ & $1-4.44$ & $0-7$ & $0-5$ \\
\hline
\end{tabular}

Correlations are Pearson coefficients except those involving \# of Suicidal Ideation or Behavior reports, which are Spearman rank correlation coefficients. ICCs are calculated on the repeated administrations of the SITBI dichotomous ideation and attempt items, CCSQ subscales, and MFQ depression

ICC Intraclass correlation coefficient

${ }^{*} p<0.05$

$* * * p<0.001$

Table 2. Predicting the presence of suicidal ideation at a given follow-up assessment from CORT AUC $\mathrm{C}_{i}$ during the TSST, person-standardized stress at the present follow-up assessment, and their interaction

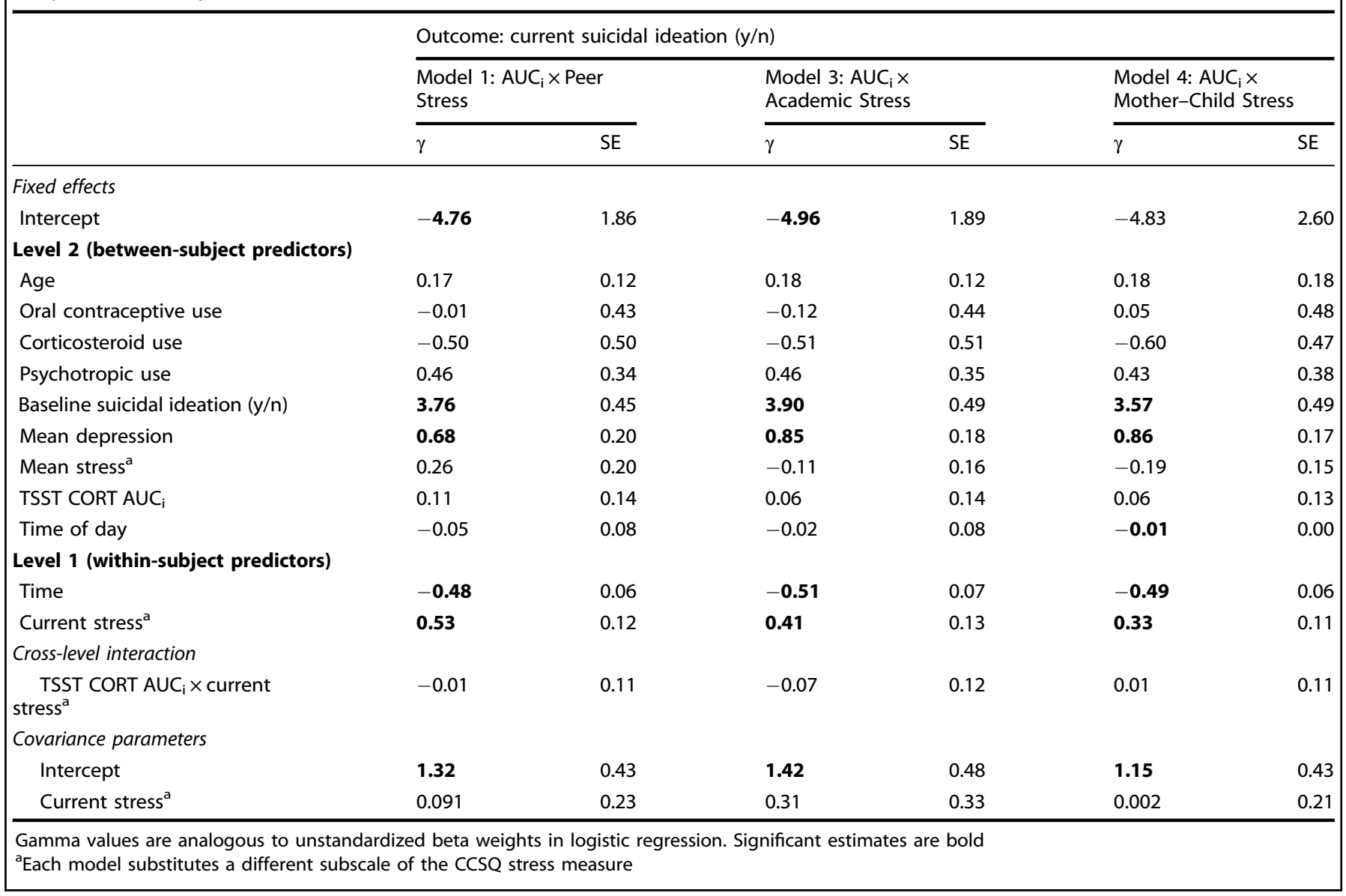


Table 3. Predicting the presence of suicidal behavior at a given follow-up assessment from CORT AUC ${ }_{i}$ during the TSST, person-standardized stress at the present follow-up assessment, and their interaction

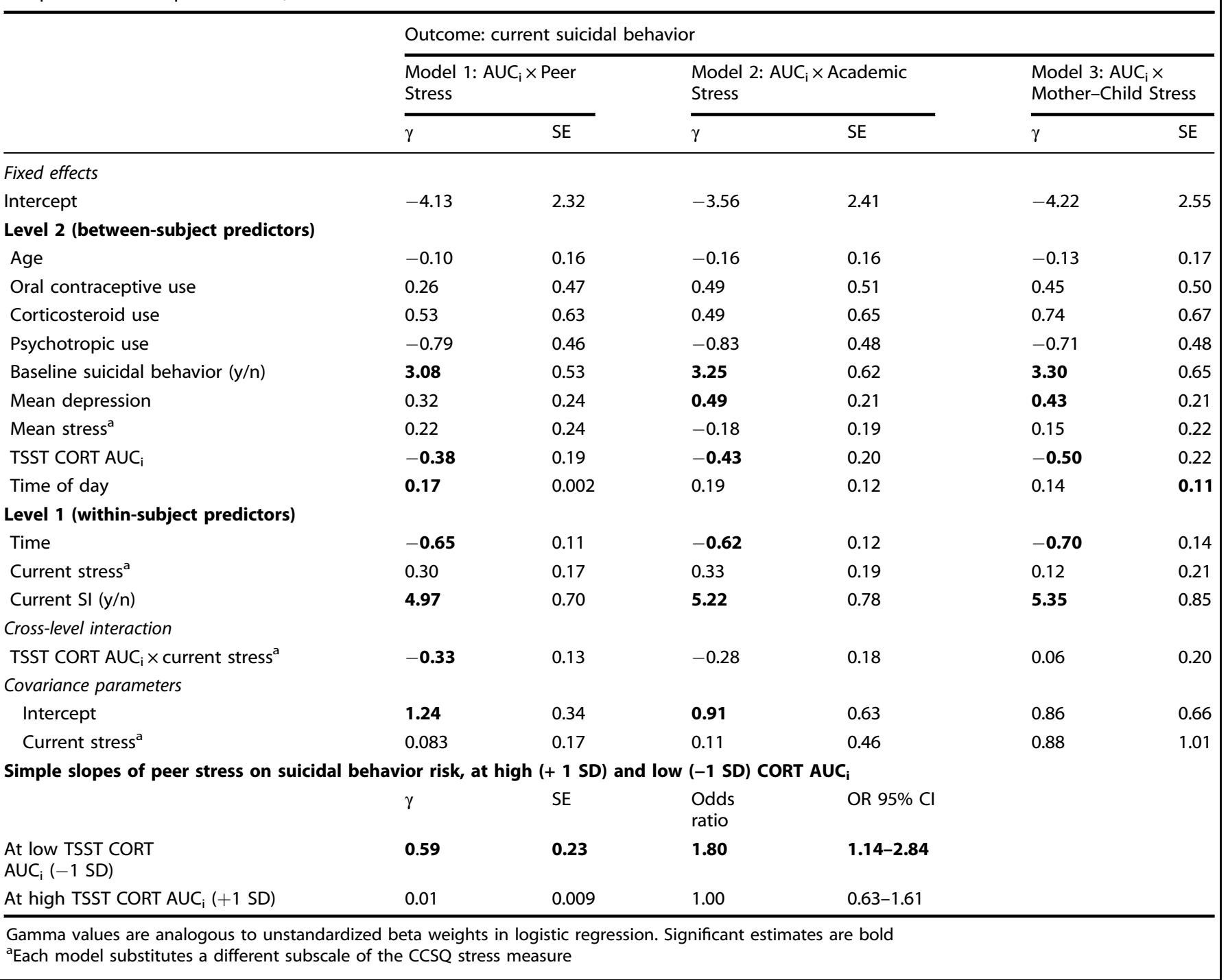

prospective within-person link between higher-than-usual stress and suicidal ideation and behavior, and that these interactions would be particularly strong for the developmentally salient peer stress predictor. Models predicting suicidal ideation from covariates, current stress (peer, academic, and mother-child), CORT $A \cup C_{i}$, and their interactions are presented in Table 2. Risk of suicidal ideation went down over time, consistent with regression to the mean following recruitment of an at-risk sample. Neither rectilinear nor quadratic terms for CORT $A U C_{i}$ predicted overall suicidal ideation. Higher-than-usual stress in each domain (peer, mother-child, and academic) was linked with greater concurrent risk of ideation (see Table 2). CORT AUC $C_{i}$ did not moderate these relationships. Therefore, stressors predicted suicidal ideation regardless of CORT response.

Models predicting suicidal behavior (over and above current ideation) from covariates, current stress (peer, academic, and mother-child), CORT $A \cup C_{i}$, and their interactions are presented in Table 3. Risk of suicidal behavior decreased over time, consistent with regression to the mean following recruitment of an at-risk sample. Higher CORT AUC $C_{i}$ predicted lower overall risk of suicidal behavior. There was no main or interactive effect of a quadratic $A \cup C_{i}$ on suicidal behavior. No main effects of stressors on behavior were observed. As predicted, CORT $A U C_{i}$ moderated the prospective association between elevated peer stress (and not the less developmentally salient academic or mother-child stress) with concurrent suicidal behavior. Simple slopes (Table 3) of peer stress predicting suicidal behavior at high vs. low CORT $A U C_{i}$ suggest that higher-than-usual peer stress predicts greater risk of behavior only among subjects with blunted/lower (-1 SD) CORT $\mathrm{AUC}_{\mathrm{i}}$; in subjects with high (+1 SD) CORT AUC $C_{\text {, }}$, higher-than-usual stress did not predict behavior. Quadratic effects of CORT $A U C_{i}$ were not found (neither main nor interactive effects of quadratic term were significant). Therefore, CORT reactivity to psychosocial stress shaped the prospective covariation of real-life peer stress with risk of suicidal behavior over and above any association with ideation (Fig. 2).

Supplemental analyses focused on (1) inclusion of abuse history and PTSD symptoms as covariates, (2) substitution of $A U C_{g}$ for $A U C_{i}$, and (3) potential quadratic effects of $A U C_{i}$ on baseline reports of lifetime suicidal ideation and behavior are presented in Supplement 2.

\section{DISCUSSION}

Using a multilevel, prospective diathesis-stress framework, we found that (1) blunted laboratory HPA axis stress responses 
Blunted CORT Reactivity Strengthens the Link Between Peer Stress and Suicidal Behavior
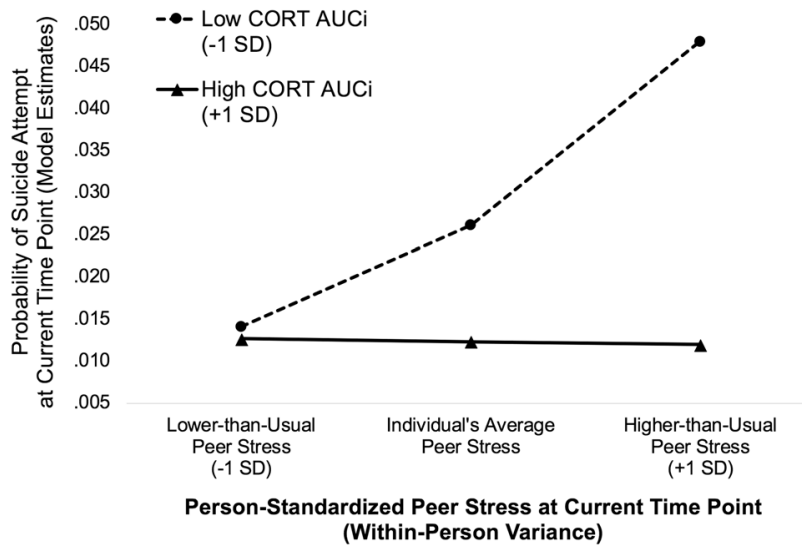

Fig. 2 Model-based estimates depicting the interaction between cortisol reactivity to the TSST $\left(A \cup C_{i}\right)$ and current level of peer stress predicting suicidal behavior at the concurrent time point

prospectively predict suicidal behavior (across 18 months) over and above current suicidal ideation, current depression, and lifetime history of suicidal behavior, and (2) that periods of elevated peer stress increase risk of suicidal behavior only among subjects with blunted CORT responses. The blunted CORT responses observed in adults with suicidal behavior $[10,11]$ may be observable in adolescent females. Consistent with adult work, this risk was specific to suicidal behavior; subjects with ideation but not suicidal behavior did not demonstrate blunted CORT responses relative to controls. Longitudinally, elevated peer stress signaled acute risk of suicidal behavior only in subjects with this blunted HPA biomarker, providing support for an integrated multilevel model of adolescent suicide risk that considers chronic biological diathesis in concert with developmentally salient environmental stressors. When subjects were grouped into suicidality histories based on all available data (no history of ideation or behavior, history of ideation only, history of behavior), individuals with histories of behavior showed a blunted CORT $A U C_{i}$ relative to those with no history of suicidality, whereas those with histories of ideation only did not. Furthermore, we observed no significant differences in CORT $A U C_{i}$ between those with histories of suicidal ideation and those with no histories of suicidality. No differences in baseline CORT were observed between groups.

The present findings extend two other studies demonstrating that blunted CORT in the context of psychosocial stressors predicts suicidal behavior [10, 11]. Crucially, this study additionally demonstrates that HPA axis response serves as a prospective predictor of suicidal behaviors, that this previously-described risk is observable in adolescent females, and that this risk of suicidal behavior among those with blunted HPA responses to stress may be observable during naturally-occurring psychosocial stress. Therefore, the present study adds to a growing literature linking altered HPA axis regulation to suicidal behavior.

In our prior analyses using this sample, we identified a significant interaction between lifetime abuse and increased risk of suicidal behavior at follow-up timepoints characterized by higher-than-usual depression [33]. Although an association between abuse history and blunted CORT response might be expected on the basis of previous work, neither history of abuse nor number of PTSD symptoms endorsed were significantly associated with CORT AUC $C_{i}$ in our sample (Supplement 1). However, covarying either abuse or number of PTSD symptoms did slightly reduce the strength of the interaction between $A U C_{i}$ and peer stress in predicting suicidal behavior (Supplement 2). In future work, both diagnostic and dimensional measures of trauma exposure and PTSD should be utilized, and these factors should be explored as potential instigators of the link between HPA axis function and suicide risk. In sum, although traumatic life events have been associated with both increased and decreased HPA axis responses to stress [34], the present study suggests the possibility that those who do have trauma-related blunting of the HPA axis may be at risk of stress-related suicidal behaviors.

Cognitive dysfunction may be one mechanism by which blunted CORT response to stress increases risk for suicidal behavior during peer stress. Moderate CORT benefits behavioral inhibition during stress [20], whereas blunted CORT responses to psychosocial stress compromise executive functioning, including emotion processing and inhibition of prepotent responses [35, 36]. Blunted CORT response to the TSST has also been found in hyperactive-impulsive type ADHD [37], a disorder characterized by poor inhibition. During peer stress, blunted CORT responses may permit failures of executive functions critical for inhibiting suicidal urges. Studies must determine whether failures of executive functions or decreased control of urges during peer stress might account for our findings.

Blunted CORT responses to psychosocial stress might promote suicidal behavior by impairing glucocorticoid regulation of inflammatory immune signaling. Social threats are accompanied by inflammatory brain signaling that is normatively downregulated by CORT at the glucocorticoid receptor (GR). If CORT stress response is compromised, perhaps due to a history of extreme stress exposures, this may permit uncontrolled inflammatory brain signaling, which increases a variety of suicide-promoting emotional experiences, including social loss, shame, and anhedonia [38]. This possibility is bolstered by evidence from Melhem et al. [10] showing adults with suicidal behavior-but not individuals with ideation-had lower hair CORT but also elevated inflammation (CRP, TNF- $a$, stimulated IL-6 production) and poorer GR sensitivity (ability of CORT to downregulate inflammation). Future studies should investigate whether blunted CORT stress response in adolescent females cause increased inflammatory brain signaling, and what role this may play in stress-related suicidal desire and action.

Although all stressors predicted ideation, only peer stress predicted suicidal behavior in adolescents with blunted CORT responses. This suggests that developmentally salient social stressors lie at the heart of risk for suicidal behavior in adolescent females [4]. Stressful peer environments may be critical risk factors as females undergo the pubertal transition, which is marked by increases in peer stress, especially for girls [39]. Progesterone and its neurosteroid metabolites, which rise and begin to fluctuate during puberty, upregulate social behaviors [40], increase social threat monitoring [41, 42], and regulate the HPA axis [43]. Pubertal ovarian steroid changes may therefore contribute to the development of stress-related suicidality.

\section{Clinical implications}

Adolescent females with suicidality may require multifaceted treatment targeting behavioral skills deficits and physiologic vulnerabilities. Skills-based psychotherapies such as dialectical behavior therapy (DBT), focused on effective behavioral responses to painful socioemotional experiences, may enhance inhibitory control and reduce suicide risk during peer stress [44]. DBT is adapted for adolescents [45] and has received promising support for reducing adolescent suicidal ideation and self-injury in a randomized controlled trial [46]. Pharmacologic treatments may also serve to reduce the deleterious impact of psychosocial stressors on depression (e.g., Normann et al. [47]); however, no evidence-based pharmacologic approaches for stress-related suicidality exist. Future work must determine whether pharmacologic stabilization or augmentation of the HPA axis might reduce suicide attempt risk in the context of stress. 
Strengths, limitations, and future directions

This study had many strengths, including prospective evaluation of suicidal outcomes, measurement at multiple Research Domain Criteria (RDoC) levels in a transdiagnostic sample [48], and the dynamic, multilevel statistical approach allowing for prospective modeling of diathesis and stress interplay. The use of a single measure of HPA axis stress response is a limitation; next steps include more frequent measures of HPA function. The correlational nature of our study is a limitation when considering causality; although likely to prove challenging, controlled experiments would allow for the evaluation of CORT (vs. placebo) effects on neurobiological function, experiences, and suicidal planning or intent while maintaining participant safety. Work is also needed to clarify whether HPA axis function and stress play a role in adolescent male suicidality. Finally, one should be cautious in generalizing findings to specific clinical groups, since various suicidality subtypes with differing pathophysiology are hypothesized. While a stress-related subtype of suicidality (characterized broadly by episodes of suicidality following stress, trauma histories, and impulsivity [49]) may be expected to demonstrate blunted CORT responses, other subtypes may have different characteristics.

\section{CONCLUSIONS}

Research on the development of suicidal behavior in adolescent females has been limited by cross-sectional approaches that do not accurately model the interactive, multilevel nature of suicide risk as it unfolds over time. Work described here highlights the complex relationships among the HPA axis response to stress, developmentally salient psychosocial stressors, and suicidal behavior in adolescent females. Specifically, peer stress appears to be prospectively linked with acute risk of suicidal behavior, but only among subjects with blunted HPA axis responses to psychosocial stress. Additional studies delineating the neurobiological and psychosocial pathways underlying these associations will be necessary to address the rising rates of suicide deaths in this highly vulnerable group.

\section{ACKNOWLEDGEMENTS}

This research was supported by grants from the National Institute of Mental Health (R01MH085505, R01MH107479, K99MH109667, and F32MH108238).

\section{ADDITIONAL INFORMATION}

Supplementary Information accompanies this paper at (https://doi.org/10.1038/ s41386-018-0206-6).

Competing interests: The authors declare no competing interests.

Publisher's note: Springer Nature remains neutral with regard to jurisdictional claims in published maps and institutional affiliations.

\section{REFERENCES}

1. Curtin SC, Warner M, Hedegaard H. Increase in suicide in the United States, 1999-2014. NCHS Data Brief. 1-8.

2. Hawton K, Fagg J, Simkin S. Deliberate self-poisoning and self-injury in children and adolescents under 16 years of age in Oxford, 1976-1993. Br J Psychiatry. 1996;169:202-8.

3. Hawton K, Saunders KEA, O'Connor RC. Self-harm and suicide in adolescents. Lancet. 2012;379:2373-82.

4. King $C A$, Merchant $C R$. Social and interpersonal factors relating to adolescent suicidality: a review of the literature. Arch Suicide Res. 2008;12:181-96.

5. Kosky R, Silburn S, Zubrick S. Symptomatic depression and suicidal ideation. A comparative study with 628 children. J Nerv Ment Dis. 1986;174:523-8.

6. Reznikoff PTM. Perceived peer and family relationships, hopelessness and locus of control as factors in adolescent suicide attempts. Suicide Life Threat Behav. 1982;12:141-50.
7. Koss KJ, Gunnar MR. Annual Research Review: early adversity, the hypothalamic-pituitary-adrenocortical axis, and child psychopathology. J Child Psychol Psychiatry. 2018;59:327-46.

8. Gunnar M, Quevedo K. The neurobiology of stress and development. Annu Rev Psychol. 2007;58:145-73.

9. Giletta M, Calhoun CD, Hastings PD, Rudolph KD, Nock MK, Prinstein MJ. Multilevel risk factors for suicidal ideation among at-risk adolescent females: the role of hypothalamic-pituitary-adrenal axis responses to stress. J Abnorm Child Psychol. 2015;43:807-20.

10. Melhem NM, Keilp JG, Porta G, Oquendo MA, Burke A, Stanley B, et al. Blunted HPA axis activity in suicide attempters compared to those at high risk for suicidal behavior. Neuropsychopharmacology. 2016;41:1447-56.

11. O'Connor DB, Green JA, Ferguson E, O'Carroll RE, O'Connor RC. Cortisol reactivity and suicidal behavior: investigating the role of hypothalamic-pituitary-adrenal axis responses to stress in suicide attempters and ideators. Psychoneuroendocrinology. 2017;75:183-91.

12. Kaess $M$, Hille M, Parzer $P$, Maser-Gluth $C$, Resch F, Brunner R. Alterations in the neuroendocrinological stress response to acute psychosocial stress in adolescents engaging in nonsuicidal self-injury. Psychoneuroendocrinology. 2012;37:157-61.

13. Plener PL, Zohsel K, Hohm E, Buchmann AF, Banaschewski T, Zimmermann US, et al. Lower cortisol level in response to a psychosocial stressor in young females with self-harm. Psychoneuroendocrinology. 2017;76:84-7.

14. Koss KJ, Cummings EM, Davies PT, Cicchetti D. Patterns of adolescent regulatory responses during family conflict and mental health trajectories. J Res Adolesc. 2017;27:229-45.

15. Lopez-Duran NL, Kovacs M, George CJ. Hypothalamic-pituitary-adrenal axis dysregulation in depressed children and adolescents: a meta-analysis. Psychoneuroendocrinology. 2009;34:1272-83.

16. Luo H, Hu X, Liu X, Ma X, Guo W, Qiu C, et al. Hair cortisol level as a biomarker for altered hypothalamic-pituitary-adrenal activity in female adolescents with posttraumatic stress disorder after the 2008 Wenchuan Earthquake. Biol Psychiatry. 2012;72:65-9.

17. Pfeffer $C R$, Altemus $M$, Heo $M$, Jiang $H$. Salivary cortisol and psychopathology in children bereaved by the September 11, 2001 terror attacks. Biol Psychiatry. 2007;61:957-65.

18. Lovallo WR. Early life adversity reduces stress reactivity and enhances impulsive behavior: implications for health behaviors. Int J Psychophysiol. 2013;90:8-16.

19. Popma A, Doreleijers TAH, Jansen LMC, Van Goozen SHM, Van Engeland $H$, Vermeiren R. The diurnal cortisol cycle in delinquent male adolescents and normal controls. Neuropsychopharmacology. 2007;32:1622-8.

20. Wingenfeld K, Wolf OT. Effects of cortisol on cognition in major depressive disorder, posttraumatic stress disorder and borderline personality disorder-2014 Curt Richter Award Winner. Psychoneuroendocrinology. 2015;51:282-95.

21. Hoyt LT, Zeiders KH, Ehrlich KB, Adam EK. Positive upshots of cortisol in everyday life. Emotion. 2016;16:431-5.

22. Reynolds CR, Kamphaus RW, Vannest KJ. Behavior assessment system for children (BASC). InEncyclopedia of clinical neuropsychology 2011 (pp. 366-371). Springer, New York, NY.

23. Allen AP, Kennedy PJ, Cryan JF, Dinan TG, Clarke G. Biological and psychological markers of stress in humans: focus on the Trier Social Stress Test. Neuroscience \& Biobehavioral Reviews. 2014 Jan 1;38:94-124.

24. Pruessner JC, Kirschbaum C, Meinlschmid G, Hellhammer DH. Two formulas for computation of the area under the curve represent measures of total hormone concentration versus time-dependent change. Psychoneuroendocrinology. 2003;28:916-31.

25. Nock MK, Holmberg EB, Photos Vl, Michel BD. Self-injurious thoughts and behaviors interview: development, reliability, and validity in an adolescent sample. Psychol Assess. 2007;19:309-17.

26. Sheehan DV, Sheehan KH, Shytle RD, Janavs J, Bannon Y, Rogers JE, et al. Reliability and validity of the Mini International Neuropsychiatric Interview for Children and Adolescents (MINI-KID). J Clin Psychiatry. 2010;71:313-26.

27. Costello EJ, Angold A. Scales to assess child and adolescent depression: checklists, screens, and nets. J Am Acad Child Adolesc Psychiatry. 1988;27:726-37.

28. Rudolph KD, Kurlakowsky KD, Conley CS. Developmental and social-contextual origins of depressive control-related beliefs and behavior. Cogn Ther Res. 2001;25:447-75.

29. Bolger N, Laurenceau JP. Intensive longitudinal methods. New York, NY: Guilford; 2013.

30. Little RJA. A test of missing completely at random for multivariate data with missing values. J Am Stat Assoc. 1988;83:1198-202.

31. Faul F, Erdfelder E, Lang A-G, Buchner A. G*Power 3: a flexible statistical power analysis program for the social, behavioral, and biomedical sciences. Behav Res Methods. 2007;39:175-91. 
32. Snijders T, Bosker R. Multilevel modeling: An introduction to basic and advanced multilevel modeling.

33. Miller AB, Eisenlohr-Moul T, Giletta M, Hastings PD, Rudolph KD, Nock MK, et al. A within-person approach to risk for suicidal ideation and suicidal behavior: examining the roles of depression, stress, and abuse exposure. J Consult Clin Psychol. 2017;85:712-22.

34. Miller GE, Chen E, Zhou ES. If it goes up, must it come down? Chronic stress and the hypothalamicpituitary-adrenocortical axis in humans. Psychological bulletin. 2007;133:25.

35. McGirr A, Diaconu G, Berlim MT, Pruessner JC, Sablé R, Cabot S, et al. Dysregulation of the sympathetic nervous system, hypothalamic-pituitary-adrenal axis and executive function in individuals at risk for suicide. J Psychiatry Neurosci. 2010;35:399.

36. Turecki G, Ernst C, Jollant F, Labonté B, Mechawar N. The neurodevelopmental origins of suicidal behavior. Trends Neurosci. 2012;35:14-23.

37. West $D$, van, Claes S, Deboutte D. Differences in hypothalamic-pituitary-adrenal axis functioning among children with ADHD predominantly inattentive and combined types. Eur Child Adolesc Psychiatry. 2009;18:543-53.

38. Slavich GM, Irwin MR. From stress to inflammation and major depressive disorder: a social signal transduction theory of depression. Psychol Bull. 2014;140:774-815.

39. Hankin BL, Mermelstein R, Roesch L. Sex differences in adolescent depression: stress exposure and reactivity models. Child Dev. 2007;78:279-95.

40. Koonce CJ, Frye CA. Progesterone facilitates exploration, affective and social behaviors among wildtype, but not 5a-reductase Type 1 mutant, mice. Behav Brain Res. 2013;253:232-9.
41. Maner JK, Miller SL. Hormones and social monitoring: menstrual cycle shifts in progesterone underlie women's sensitivity to social information. Evol Hum Behav. 2014;35:9-16.

42. Wingen GA, van, Broekhoven $F$, van, Verkes RJ, Petersson KM, Bäckström $T$, Buitelaar JK, et al. Progesterone selectively increases amygdala reactivity in women. Mol Psychiatry. 2008;13:325-33.

43. Roca CA, Schmidt PJ, Altemus M, Deuster P, Danaceau MA, Putnam K, et al Differential menstrual cycle regulation of hypothalamic-pituitary-adrenal axis in women with premenstrual syndrome and controls. J Clin Endocrinol Metab. 2003;88:3057-63.

44. Linehan M. DBT skills training manual. Guilford Publications; 2014.

45. Rathus JH, Miller AL. DBT ${ }^{\circledR}$ skills manual for adolescents. Guilford Publications; New York, NY. 2014.

46. Mehlum L, Tørmoen AJ, Ramberg M, Haga E, Diep LM, Laberg S, et al. Dialectical behavior therapy for adolescents with repeated suicidal and self-harming behavior: a randomized trial. J Am Acad Child Adolesc Psychiatry. New York, NY. 2014;53:1082-91.

47. Normann C, Frase S, Haug V, von Wolff G, Clark K, Münzer P. et al. Antidepressants rescue stress-induced disruption of synaptic plasticity via serotonin transporterindependent inhibition of L-type $\mathrm{Ca}^{2+}$-channels. Biol Psychiatry. 2018;84:55-64.

48. Glenn CR, Cha CB, Kleiman EM, Nock MK. Understanding suicide risk within the Research Domain Criteria (RDoC) Framework: insights, challenges, and future research considerations. Clin Psychol Sci. 2017;5:568-92.

49. Bernanke JA, Stanley BH, Oquendo MA. Toward fine-grained phenotyping of suicidal behavior: the role of suicidal subtypes. Mol Psychiatry. 2017;22:1080-1. 